\title{
Research on Face Recognition of Large Capacity Coherent Photoelectric Hybrid ZHOU Yan-min ${ }^{1, a}$, LI Jian-fang ${ }^{1, b}$ \\ ${ }^{1 .}$ Chongqing College of Electronic Engineering, Chongqing 401331, China \\ a zhouyanmin023@126.com, ${ }^{b} 444632332 @ q q . c o m$
}

\section{Keywords: Large Capacity Coherent Light; Face Recognition; Photoelectric Mixing}

\begin{abstract}
With the increase of the face database, the electronic computing is exponentially increasing, and the traditional face recognition method can't meet the requirement of real-time recognition. Optical actuator have parallel processing, fast features, and thus in the face recognition side and presents a huge potential. The application of photoelectric hybrid processing system in face recognition should not only follow the common law of ordinary photoelectric hybrid processing system, but also play the advantages of light calculation. The application of photoelectric hybrid processing system in human visual target recognition is to follow the common law of ordinary photoelectric hybrid processing system, but also take into account the special needs of human visual application, so its feasibility study status, research level, photoelectric hybrid processing A large number of studies selected to improve the innovation suitable for robot vision recognition hardware structure and software algorithms and other issues to explore the issue.
\end{abstract}

\section{Theoretical Introduction of Coherent Light}

Coherent light refers to all the parameters that can be predicted and correlated at any point in time or space, especially in a region on a plane perpendicular to the propagation direction, or at a particular point in space. The working principle of the system is more close to that of conventional radio communication systems than conventional optical fiber communication systems. In the coherent optical fiber communication system, the digital signal adopts amplitude modulation keying (ASK), frequency shift keying (FSK) and phase shift keying (PSK). In other word, the scene must be processed with a further digital algorithm to reconstruct the $3-\mathrm{D}$ image in the computer's memory.

High sensitivity: coherent demodulation of the signal amplitude and the local oscillator amplitude is proportional to. Since the power of the local oscillator source is much greater than the signal power, the resulting signal amplitude will be much larger than the direct detection. The photo detection quantum noise is also scaled up, and the received signal-to-noise ratio is greatly improved due to the constant thermal noise amplitude of the photo detector and the receiver, which in turn improves the reception sensitivity. In addition, the use of frequency shift keying or phase shift keying of these excellent modulation can also make the sensitivity to further improve ${ }^{[1]}$.

Generally, the reception sensitivity of the coherent receiving system can be $10 \sim 20 \mathrm{~dB}$ higher than that of the conventional optical fiber communication system. The selectivity is good: the conventional optical fiber communication system is multiplexed and must be optically filtered. Similar to the radio communication system, the local oscillator and the signal light are subjected to an intermediate frequency in the coherent optical communication system, and an electrical filter can be used at the intermediate frequency. Since the electrical filter is much more selective than the optical filter, the coherent receiving system has excellent selectivity. It can make full use of quartz fiber low loss window, and achieve frequency division multiplexing and high density wavelength division multiplexing multi-channel transmission. In the visual system, many cases can be identified by binary images, but gray-scale image recognition is versatile. 


\section{Principle of Coherent Light Processing Algorithm}

In the recognition system based on coherent optical actuator, the generalized complementary coding is not used for the image, but the gray scale coding of the original image is used to improve the discrimination ability of optical correlation recognition. First, the original image is gray scale equalized, so that the gray scale is evenly distributed between 0 and 255, and 256 gray levels are divided into four regions $(0 \sim 63,64 \sim 127,128 \sim 191,192 \sim 255)$, and use the four regions to divide the original gray scale graph (the gray value of the gray value falls within the region becomes 0 , the other point becomes 255, and vice versa), Generate four binary images. And then 4 images and then connected to an image ${ }^{[2]}$. Fig.1 shows the Overall structure of electronic hybrid matched filtering actuator.

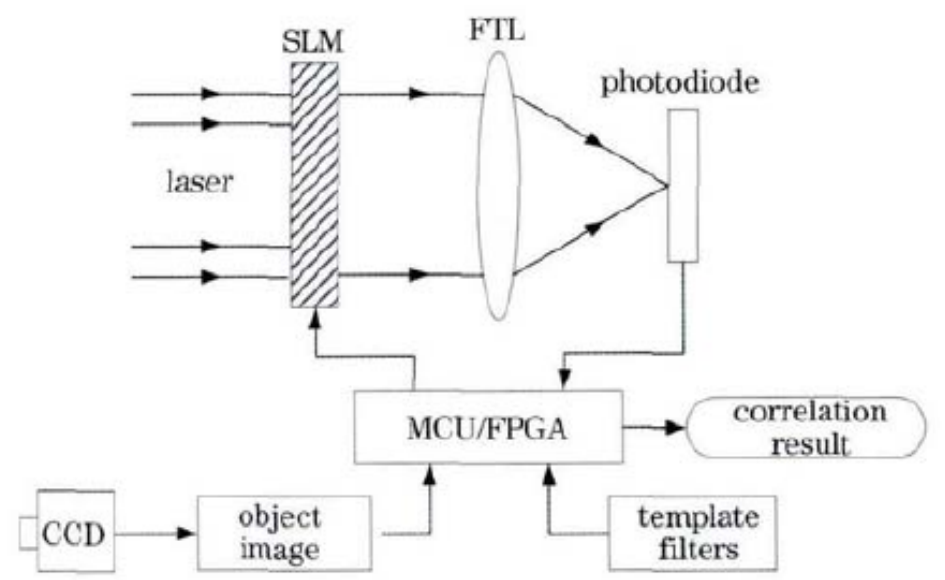

Fig. 1.The Overall structure of electronic hybrid matched filtering actuator

The advantage of this coding method is that: 1) play a similar role with the generalized complementary code, the use of optical system step by step to achieve mathematical morphology hit in the transformation, so that the image of black and white pixels proportional balance, so that the image background and prospects related 2) The optical operation quality and the anti-illumination ability of the gray scale image are not as good as the binary image, so the gray image becomes binary image and the optical operation is favorable. 3) In the generalized complementary coding, one gray-scale map through a secondary domain binarization, and form four different domain binarization, retain more of the original gray-scale information.

After encoding the image, the width and height of the image are doubled, but the aspect ratio of the original image is maintained, and the coding pattern of the square $(256 \times 256$ pixds $)$ can take full advantage of the recognition capability of the original system. In this paper, we use 1050 face as the original image library and extract the feature through KL transformation. After reconstruction verification and experimental verification, we choose the eigenvector corresponding to the first 16 largest eigenvalues and visualize it as the face library. On the face, gray scale equalization and coding can improve the identification of optical correlation recognition ${ }^{[3]}$.

\section{Visual Recognition Level of Photoelectric Hybrid System}

Optoelectronic hybrid system hardware structure trade-off principle should be: low cost, high computational speed volume should not be too large, the device is easy to buy the best commercial structure is simple and reliable, and with CCD as a square detection device to record the input signal combined transform power spectrum, The detected graphics and then through the SLM display, and use the CCD to detect the relevant output. The hardware structure has a low price, compact, intuitive to reflect the basic principles of JT C, for future exploration and innovation is more suitable for robot vision JT C hardware Structure to provide the basis ${ }^{[5]}$.

In robot vision, there are two basic preprocessing methods, the first is based on the method of 
spatial domain technology, the second is based on Fourier transform frequency domain technology method, due to the need for a large amount of calculation in the robot vision The frequency domain method in the system is far less common than the spatial domain method, but if the JT C platform is used, the two-dimensional Fourier transform can be calculated at the speed of light to provide a powerful light for the preprocessing method using frequency domain techniques in robot vision. It can be seen that the pretreatment of frequency domain preconditioning with JTC is a kind of enhancement and expansion of computer pretreatment ability for robot vision pretreatment problem. Gray-scale systems can also function if they can't achieve good contrast and therefore do not facilitate the use of binary image systems.

The three-dimensional optical actuator provides the possibility of direct and fast processing of images, similar to the fast and parallel way of 2D actuator. 3D targets can be identified and tracked by $3 \mathrm{D}$ optical actuator. 3-D optical correlation basic algorithm is that the 3-D observation scene and the reference object are first mapped side by side to the 2-D plane, and then the traditional 2-D optical correlation is carried out. However, the distribution of the observed scene must be the first. This method is used in conjunction with the three-dimensional recognition of the electronic calculation methods are very similar, which are based on the reconstruction of three-dimensional images. Morphological correlation introduced pattern recognition, the basic algorithm is to first draw the gray-scale input image and gray-scale reference image into many image slices, and then in the input and reference images between the relevant operations, and finally summed up all the results related output to obtain morphological correlation.

\section{Face Recognition System}

Combining the visual spatial domain method and the frequency domain method with JTC, JTC, JTC and JTC were used to simulate the face computer. First of all, the use of classic JTC for face image recognition, when the two face image itself or its edge images are similar, the classic JTC face recognition effect is poor. The JTC based on spatial domain method is used to identify the face target. As the face image is fine and complicated, some edge detection algorithms have poor effect on the edge of the human face, so that the face is deformed by the edge detection, or the edge feature of the face is not enough, so it can't achieve the purpose of improving the recognition.

Image contour extraction algorithm Yan to extract the edge of the face, the essence of the algorithm lies in the hollowing out the internal point, a pixel for the boundary pixel condition if and only if its value is 1 and at least one value of 0 neighborhood pixels. You can use 4 connected neighbors or 8 connected neighbors. This is actually equivalent to using a 4-connected neighborhood or 8 connected to the structure of the neighborhood to check the original image corrosion, and then subtract the original image corrosion image. Based on the 4-connected neighborhood binary image contour extraction algorithm, the face shape feature of the face image is extracted, and the face recognition is performed by the classical JTC. The recognition effect is improved, as shown in the figure. Fig. 2 shows the identify output peaks for different faces.

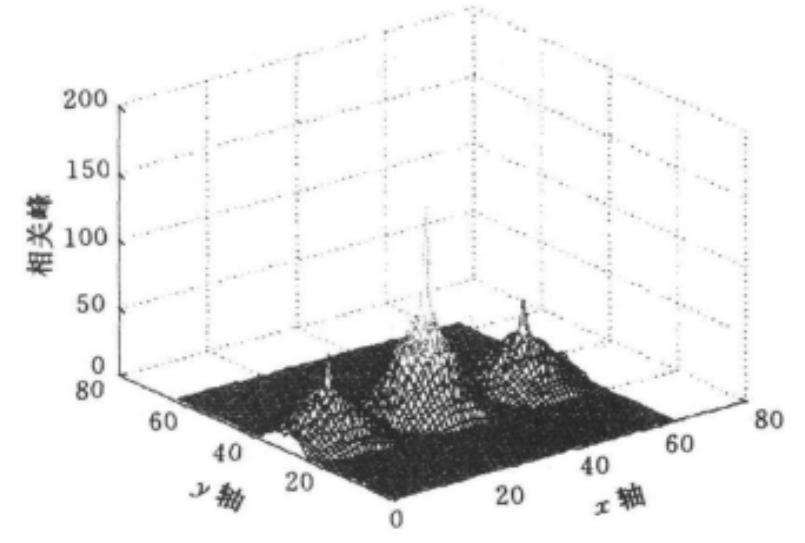

Fig. 2.The Identify output peaks for different faces

Through the above analysis shows that the use of spatial domain detection and pretreatment, 
JTC face recognition effect has improved. In order to further improve the recognition, the threshold algorithm based on frequency domain method preprocessing is combined with JTC for face recognition. The non - linear binary JTC for preprocessing of the spectral surface is introduced, and the power spectrum based on the frequency domain is preprocessed. The ICJ power spectrum is obtained by the value of the input image and the reference image power spectrum. Only a part of the common image processing algorithm can be used in robot vision. By combining the classical preprocessing algorithm suitable for robot vision and the algorithm equivalent to its computational complexity and JTC, the robot visual target can be identified, especially the JTC photoelectric hybrid processing system is obtained by light calculation Input field power spectrum, which solves the bottleneck problem of robot visual frequency domain method pretreatment, making it possible to preprocess the spatial domain method and frequency domain method.

\section{Conclusions}

Based on the coherent optical photoelectric hybrid recognition system, the KL transform is used to extract and extract the image features. Through the specific algorithm, a large number of large-capacity face retrieval and recognition systems are realized and the speed and accuracy are achieved. As the JT C photoelectric hybrid processing system uses the optical calculation to obtain the power spectrum based on the frequency domain, it solves the bottleneck problem of large face recognition and provides the possibility of further preprocessing the frequency domain method. When the number of library graphs increases linearly, the number of feature faces used to identify the feature after the feature extraction only needs a small increase to meet the requirement of recognition, and it can show that the image retrieval can be realized when the recognition time is very small.

\section{References}

[1] Eriko Watanabe, Kashiko Kodate. Fast face-recognition optical parallel correlator using high accuracy correlation filter [J]. Opt Rev. 2005, LZ (6):460-46 6.

[2] Eriko Watanabe, Kashiko Kodate. High speed holographic optical correlator or face recognition Mario I, C'hacon M. State of the Art in Face Recognition [M]. Vienna: I-Tech, 2009, 109-130.

[3] Li Jianhua, Cao Liangcai, Tan Xiaodi. Transimission type of collinear volume holographic storage technology based on LiNb0; 3 crystal [J]. Acta Optica Sinica, 2012, 32(4):0409001.

[4] Wan Yu hong, Tao Shiquan. Micro-holographic data storage technology and its research progress [J]. Laser g. Optoelectronics Progress, 2012, 49 (10):100004.

[5] Guo Suoli, Cheng Pan pan, Liu Yanfei. Large-scale face recognition using optic-electronic hybrid joint transform correlator [J].Chinese J Lasers, 2012, 39(s2):s209004. 